\title{
Effect of whitening dentifrices: a double-blind randomized controlled trial
}

\section{Karen PINTADO-PALOMINO(a) Cecília Vilela Matias VASCONCELOS ${ }^{(a)}$ Roberto Juns da SILVA(b) Andre Luiz de Macedo FRESSATT(b) Bianka Jurca Gonçalves da MOTTA ${ }^{(b)}$ Fernanda de Carvalho Panzeri PIRES-DE-SOUZA(a) Camila TIRAPELLI(a)}

anniversidade de São Paulo (USP), School of Dentistry of Ribeirão Preto, Department of Dental Materials and Prosthodontics, Ribeirão Preto, SP, Brazil.

bUniversidade de São Paulo (USP), School of Dentistry of Ribeirão Preto, Ribeirão Preto, SP, Brazil.
Declaration of Interests: The authors certify that they have no commercial or associative interest that represents a conflict of interest in connection with the manuscript.

\section{Corresponding Author:}

Camila Tirapelli

E-mail: catirapelli@forp.usp.br

DOI: 10.1590/1807-3107BOR-2016.vol30.0082

Submited: Sep 22, 2015

Accepted for publication: Mar 23, 2016

Last revision: May 09, 2016
Abstract: The aim of this clinical study was to evaluate tooth color changes $(\Delta \mathrm{E})$ and tooth sensitivity (TS) associated with whitening dentifrices. Sixty participants were selected according to inclusion and exclusion criteria and then allocated to three groups $(n=20)$ : Colgate Luminous White (G1), Close Up White Now (G2) and Sorriso dentifrice (G3-control). The participants were instructed to use only the provided dentifrice and toothbrush in standardized oral hygiene procedures for 4 weeks. $\Delta \mathrm{E}$ was assessed by spectrophotometry (CIELab System) whereas TS was determined by a visual analog scale at baseline and weekly using four assessment points. The data were analyzed by two-way ANOVA and Tukey's post-hoc test and Friedman test $(\alpha=0.05) . \Delta \mathrm{E}$ (baseline-assessment point 4 ) showed no significant difference $(\mathrm{p}>0.05)$ across the groups: $\mathrm{G} 1(\Delta \mathrm{E}=5.1), \mathrm{G} 2(\Delta \mathrm{E}=6.8)$, and $\mathrm{G} 3(\Delta \mathrm{E}=4.4)$. $\Delta \mathrm{b}$ (baseline-assessment point 4$)$ was significantly different ( $p<0.05)$ in G2 (3.8) when compared to G1 (-0.2) and G3 (0.3). There was no significant difference $(p>0.05)$ in TS at baseline. Both the control and whitening dentifrices caused similar tooth color changes $(\Delta \mathrm{E})$. There was no significant TS during the study period.

Keywords: Treatment Outcome; Color; Dentin Sensitivity; Toothpastes; Tooth Bleaching.

\section{Introduction}

Dentifrices claiming whitening properties are largely available all over the world and are the most accessible over-the-counter option to those who want to whiten their teeth, as tooth color has become an esthetic concern, especially in recent years. ${ }^{1}$ Studies have shown that dissatisfaction with discolored teeth ranges from 18 to $53 \%,{ }^{2}$ being observed in approximately $40 \%$ of individuals aged 16 to 54 years. ${ }^{1}$ In this sense, whitened teeth may influence quality of life as they improve satisfaction with dental appearance. ${ }^{3}$ There are many approaches to manage intrinsic and extrinsic tooth discoloration, including at-home and in-office tooth bleaching treatment supervised by the dentist, restorative treatment, and even prosthetic treatment.

Access to in-office treatments is restricted to part of the population. Therefore, there has been an interest in developing methods so that patients can remove stains and apply tooth whitening at home. Accordingly, dentifrices, due to their ease of use and low cost, have been used as vehicles for whitening agents. Some in vitro studies attest the effectiveness 
of whitening dentifrices ${ }^{4,5,6}$ and some published clinical trials demonstrate greater effectiveness of one or another product. ${ }^{78,9,10,11}$ In general, these studies have argued that whitening dentifrices may reduce or significantly inhibit the extrinsic staining of natural teeth compared to control dentifrices. Despite methodological differences, most studies have used the CIELab system to observe enamel color changes; however, they have not evaluated all of the available parameters. They have observed only the delta E; but considering color or even staining, the changes from red to green and from yellow to blue and in luminosity are actually important.

The CIELab color scale is based on three elements: hue, chroma, and lightness (value), in which $L^{*}$ represents lightness, and $a^{*}$ and $b^{*}$ represent the color-opponent dimensions on a red/green and yellow/blue axis, respectively. Along the $a^{*}$ axis, positive values indicate a tendency towards red and negative values a tendency towards green. Along the $b^{*}$ axis, negative values indicate a tendency towards blue and positive values a tendency towards yellow. ${ }^{12}$

This scale may be used in every object whose color can be measured. It is actually the most widely used system in different studies ${ }^{6,12,13,14}$ and it is recommended by the American Dental Association. ${ }^{15}$ Also, there are delta values associated with this scale $\left(\Delta \mathrm{L}^{*}, \Delta \mathrm{a}^{*}\right.$, and $\left.\Delta b^{*}\right)$ which indicate how the sample differs from the standard for $L^{*}, a^{*}$ and $b^{*}$ and are used to calculate total color difference $(\Delta \mathrm{E})$ by the formula $\Delta \mathrm{E}=\left[\left(\Delta \mathrm{L}^{*}\right)^{2}+\left(\Delta \mathrm{a}^{*}\right)^{2}+\left(\Delta \mathrm{b}^{*}\right)^{2}\right]^{1 / 2} .^{16}$

Whitening dentifrices include different active ingredients in their composition, such as chemical and optical agents, and normally contain a higher amount of abrasives and detergents than do conventional dentifrices. ${ }^{2,17}$ Even though chemical action is not well elucidated, abrasive components associated with tooth brushing seem to be the main factor leading to the tooth whitening effect as a result of stain removal. ${ }^{6,18,19}$ In this sense, it is important to point out that dentifrices with higher amounts of abrasive may produce increased surface roughness in dental tissues ${ }^{20,21}$ or restorations, ${ }^{22}$ dentin abrasion, ${ }^{18}$ or even tooth sensitivity, ${ }^{23}$ especially if they are used routinely.
In this way, our research hypothesis is that two commercially available whitening dentifrices are efficient because they change tooth color without causing side effects (e.g., tooth sensitivity - TS). To test this hypothesis, Colgate Luminous White ${ }^{\circledR}$ (Colgate-Palmolive Company, São Paulo, Brazil), Close Up White Now ${ }^{\circledR}$ (Unilever, São Paulo, Brazil), and Sorriso ${ }^{\circledR}$ (Colgate-Palmolive Company, São Paulo, Brazil) dentifrices were clinically evaluated and compared as to their capacity of altering tooth color, using the colorimetric index according to the CIELab model and assessing tooth sensitivity by the visual analog scale (VAS).

\section{Methodology}

This was a double-blind, randomized controlled trial conducted at the School of Dentistry of Ribeirão Preto, University of São Paulo, Brazil, following the guidelines published by Consolidated Standards of Reporting Trials (CONSORT). The study was approved by the local Ethics Committee (Protocol no. 01546412.4.0000.5419) and complied with the Declaration of Helsinki. The clinical trial was registered at the U.S. National Institutes of Health (ClinicalTrials.gov) \#NCT02483013.

The sample size was calculated by the G Power software. ${ }^{24}$ With a $95 \%$ confidence interval, an $80 \%$ power, and an effect size ${ }^{2}$ of $80 \%$, a sample size of $n=60$ (20 in each group) was necessary.

Subjects who desired to participate in the study were examined and selected according the following inclusion criteria: (a) age over 18 years, (b) at least six anterior teeth without restorations, and (c) good general and oral health (no dental caries and periodontal disease). The exclusion criteria were: (a) tooth sensitivity, (b) any condition that could cause tooth sensitivity (non-carious cervical lesions, dentin exposure), (c) participants under orthodontic treatment, and (d) smokers.

The selected participants signed an informed consent form. Sixty eligible participants were randomly allocated, by using sequentially numbered envelopes, to one of the following three groups according to the dentifrice used ( $n=20)$ : Colgate Luminous White (G1), Close Up White Now (G2), and Sorriso (G3 - Control). Table 1 shows the experimental groups and the composition of each dentifrice. 
Table 1. Experimental groups, dentifrice and composition.

\begin{tabular}{|c|c|c|}
\hline Group & Dentifrice & Composition \\
\hline G1 & $\begin{array}{l}\text { Colgate Luminous White } \\
\text { (Colgate-Palmolive Company, } \\
\text { São Paulo, Brazil) }\end{array}$ & $\begin{array}{l}\text { Water, hydrated silica, sorbitol, pentasodium triphosphate, tetrapotassium } \\
\text { pyrophosphate, sodium lauryl sulfate, polyethylene, cocamidopropyl betaine, } \\
\text { sodium saccharin, sodium hydroxide, sodium fluoride (1 } 100 \text { ppm fluoride), } \\
\text { titanium dioxide, blue \# } 1 \text { aluminum lac. }\end{array}$ \\
\hline G2 & $\begin{array}{l}\text { Close up White Now } \\
\text { (Unilever, São Paulo, Brazil) }\end{array}$ & $\begin{array}{l}\text { Water, sorbitol, hydrated silica, PEG-32, sodium lauryl sulfate, flavor, cellulose } \\
\text { gum, sodium fluoride, sodium saccharin, PVM/MA Copolymer, glycerin, trisodium } \\
\text { phosphate, dipentene. }\end{array}$ \\
\hline $\begin{array}{l}\text { G3 } \\
\text { (Control) }\end{array}$ & $\begin{array}{l}\text { Sorriso }^{\circledR} \\
\text { (Colgate-Palmolive Company, } \\
\text { São Paulo, Brazil) }\end{array}$ & $\begin{array}{l}\text { Calcium carbonate, water, glycerin, sodium lauryl phosphate, } \\
\text { monofluorphosphate (1450 ppm), cellulose gum, pyrophosphate, sodium } \\
\text { bicarbonate, benzene alcohol sodium saccharin, sodium hydroxide. }\end{array}$ \\
\hline
\end{tabular}

One of the authors, who was not involved with the clinical period of the study, removed the contents of the dentifrices and placed them in new identical packages for the three experimental groups, and then he labeled the dentifrices with different codes; therefore, the examiners and the participants were blinded to the dentifrices being delivered.

At the beginning of the treatment period, the participants received one of the three dentifrices and a soft-bristled manual toothbrush (Johnson \& Johnson $^{\circledR}$ ). In order to standardize oral hygiene, the participants were instructed to perform three daily brushings for at least 2 to 3 minutes for 4 weeks and to use only the assigned dentifrice during that period.

Tooth color and TS were assessed at baseline (prior to the treatment) and once weekly at the 1st (t1), 2nd (t2), 3rd (t3) and 4th (t4) assessment points by two previously calibrated blinded examiners. Tooth color was measured at each visit using a Vita Easyshade spectrophotometer (VITA Zahnfabrik, BadSäckingen, Germany). Color was determined with CIEL*a* $b^{*}$ parameters. Thus, the values of $L^{*}$ (lightness), $a^{*}$ (hue on the red-green axis) and $b^{*}$ (hue on the blue-yellow axis) were recorded. In order to standardize the assessment, three measurements were taken from the middle third of the buccal surface of the right central incisor.

TS was assessed by using a visual analog scale (VAS) whose scores ranged from zero (no pain) to 10 (intolerable pain). A cold air (approximately $10^{\circ} \mathrm{C}$ ) stimulus was applied from a triple syringe placed $1 \mathrm{~cm}$ from the buccal surface in the cervical area of the selected teeth $(16,11,26,36,41$ and 46) during 3 seconds; additionally, a tactile stimulus (dental probe \# 05) was applied to the cervical region (mesial and distal areas) of each tooth. The participants recorded sensitivity perception by placing a mark on the scale.

$\Delta \mathrm{E}$ was calculated in order to compare color changes. In addition, differences at each assessment point for $\mathrm{a}^{*}, \mathrm{~b}^{*}$ and $L^{*}$ values were considered. The data were analyzed by two-way ANOVA and by Tukey's post-hoc test, using dentifrices and time as factors. To observe TS, VAS values were evaluated with Friedman test (between the three groups at the 5th assessment point). The Prism software, version 6.0 (GraphPad, CA, USA), was used to analyze the data and statistical significance was set as $\alpha=0.05$.

\section{Results}

Sixty-nine participants were screened for eligibility, of which 60 met the eligibility criteria. Approximately $67 \%$ of the selected participants were women and $33 \%$ men, with a mean age between 19 and 36 years (Table 2). At the end of 4 weeks, 50 participants completed the study. Three, four, and three participants from G1, G2 and G3, respectively, were lost to follow-up (Figure 1).

The analysis of variance for $\Delta \mathrm{E}$ revealed no statistically significant differences $(p>0.05)$ neither for the dentifrice factor nor for the assessment point factor during the study period. Despite no significant results for $\Delta \mathrm{E}$, its values were markedly high after 4 weeks, implying visible changes in tooth color in $\mathrm{G} 1(\Delta \mathrm{E}=5.1), \mathrm{G} 2(\Delta \mathrm{E}=6.8)$, and $\mathrm{G} 3(\Delta \mathrm{E}=4.4)$.

Table 2. Baseline demographic data for subjects starting the study.

\begin{tabular}{lcccc}
\hline \multirow{2}{*}{ Group } & \multicolumn{2}{c}{ Number of volunteers } & \multicolumn{2}{c}{ Age } \\
\cline { 2 - 5 } & Men & Women & Mean & Range \\
\hline G1 & 6 & 14 & 22.2 & $21-24$ \\
G2 & 8 & 12 & 23.4 & $19-36$ \\
G3 & 6 & 14 & 23.1 & $19-31$ \\
\hline
\end{tabular}




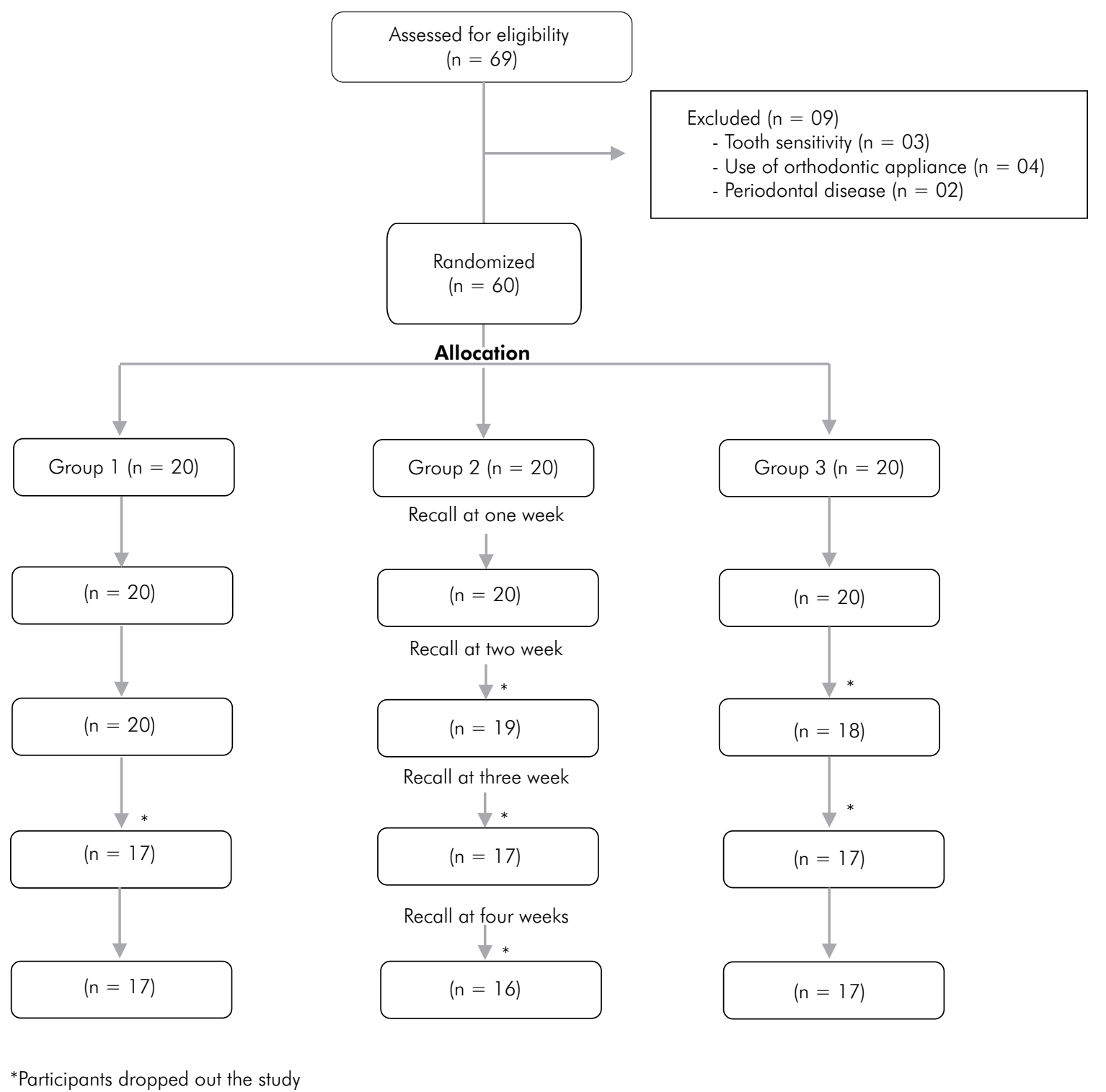

Figure 1. The consort flow chart.

Table 3 shows the values and statistically significant differences of $\Delta \mathrm{E}, \Delta \mathrm{L}, \Delta \mathrm{a}$ and $\Delta \mathrm{b}$ for each group. $\mathrm{G} 1$ participants showed significant alterations in $\Delta \mathrm{L}$ when compared to assessment points $\mathrm{t} 1-\mathrm{t} 2$ and $\mathrm{t} 2-\mathrm{t} 3$. G2 exhibited significant alteration in $\Delta$ a between $\mathrm{t} 2 \mathrm{t} \mathrm{t} 3$ and $\mathrm{t} 3-\mathrm{t} 4 . \Delta \mathrm{b}$ also showed a statistically significant difference in time and dentifrice factors in G2. Regarding time, there was a significant difference between Baseline-t4 and Baseline-t1, t1-t2 and t2-t3. Concerning dentifrices, Tukey's test showed that, after 4 weeks of treatment, $\Delta b$ was significantly different for G2 when compared with G1 and G3 (control).
TS showed no statistically significant difference $(p>0.05)$ between groups and assessment points.

\section{Discussion}

Different in vitro ${ }^{4,6,13}$ and clinical studies ${ }^{9,10,11,14,23}$ have investigated the effectiveness of whitening dentifrices and obtained contradictory results. Their efficacy has been determined by their relative ability to prevent or reduce the formation of extrinsic stains or tooth color changes over time. ${ }^{2}$ In particular, regarding tooth color change, only $\Delta \mathrm{E}$ or other parameters from the scale have been considered, frequently neglecting 
Table 3. Mean values and standard deviation of $\Delta E, \Delta a, \Delta b, \Delta L$ for each group.

\begin{tabular}{|c|c|c|c|c|}
\hline \multicolumn{2}{|c|}{ Variables } & G1 & G2 & G3 \\
\hline \multirow{5}{*}{$\Delta \mathrm{E}$} & Baseline-tl & $3,9 \pm 3,0 \mathrm{aA}$ & $5,5 \pm 2,8 a A$ & $3,8 \pm 2,6 a A$ \\
\hline & $+1-+2$ & $4,7 \pm 3,1 a A$ & $4,7 \pm 3,4 \mathrm{aA}$ & $4,1 \pm 2,5 a A$ \\
\hline & †2-†3 & $4,2 \pm 2,8 a A$ & $4,8 \pm 2,3 a A$ & $5,6 \pm 2,8 a A$ \\
\hline & $+3-+4$ & $4,5 \pm 2,9 a A$ & $5,2 \pm 3,9 a A$ & $5,4 \pm 3,7 \mathrm{aA}$ \\
\hline & Baseline- +4 & $5,1 \pm 2,8 a A$ & $6,8 \pm 3,5 a A$ & $4,4 \pm 3,0 \mathrm{aA}$ \\
\hline \multirow{5}{*}{$\Delta \mathrm{a}$} & Baseline- $\dagger 1$ & $-0,1 \pm 0,6 \mathrm{aA}$ & $0,3 \pm 1,2 a A B$ & $0,2 \pm 0,5 a A$ \\
\hline & $+1-+2$ & $0,3 \pm 0,6 a A$ & $-0,3 \pm 1,0 \mathrm{aAB}$ & $-0,1 \pm 0,6 a A$ \\
\hline & $+2-+3$ & $-0,1 \pm 0,9 a A$ & $0,5 a \pm 1,2 a A$ & $-0,2 \pm 1,1 \mathrm{aA}$ \\
\hline & $+3-\uparrow 4$ & $0,0 \pm 1,1 \mathrm{aA}$ & $-0,4 b \pm 1,2 a B$ & $-0,2 \pm 0,7 a A$ \\
\hline & Baseline- +4 & $0,1 \pm 1,0 a A$ & $0,1 \pm 0,9 a A B$ & $0,0 \pm 0,7 a A$ \\
\hline \multirow{5}{*}{$\Delta \mathrm{b}$} & Baseline-t1 & $0,0 \pm 1,0 a A$ & $-0,2 \pm 2,6 \mathrm{aB}$ & $-0,1 \pm 1,4 a A$ \\
\hline & $+1-+2$ & $0,0 \pm 1,9 a A$ & $0,2 \pm 2,6 \mathrm{aB}$ & $0,3 \pm 2,7 a A$ \\
\hline & $+2-+3$ & $0,1 \pm 1,7 a A$ & $0,8 \pm 1,9 a B$ & $0,3 \pm 3,3 a A$ \\
\hline & †-†4 & $-0,4 \pm 1,1 \mathrm{aA}$ & $2,8 \pm 3,9 a A B$ & $0,2 \pm 2,5 a A$ \\
\hline & Baseline- +4 & $-0,2 \pm 1,4 \mathrm{aA}$ & $3,8 \pm 4,1 \mathrm{bA}$ & $0,2 \pm 1,9 a A$ \\
\hline \multirow{5}{*}{$\Delta \mathrm{L}$} & Baseline- +1 & $-0,3 \pm 4,8 a A B$ & $-1,7 \pm 5,2 a A$ & $-1,7 \pm 4,0 a A$ \\
\hline & $+1-+2$ & $-2,2 \pm 4,7 \mathrm{aB}$ & $-2,0 \pm 4,7 a A$ & $-0,2 \pm 4,0 a A$ \\
\hline & $+2-+3$ & $2,4 \pm 4,0 \mathrm{aA}$ & $2,1 \pm 4,2 \mathrm{aA}$ & $0,0 \pm 5,2 a A$ \\
\hline & †3-†4 & $-0,2 \pm 5,2 a A B$ & $0,3 \pm 4,3 a A$ & $2,1 \pm 5,7 a A$ \\
\hline & Baseline- +4 & $-0,4 \pm 5,6 a A B$ & $-0,9 \pm 4,9 a A$ & $0,4 \pm 5,0 a A$ \\
\hline
\end{tabular}

Different uppercase letters indicate statistical significant difference $(p<0.05)$ within columns. Different lowercase lefters indicate statistical significant difference $(p<0.05)$ within rows.

luminosity and chromaticity. Those factors change the optical effect of tooth color; so, even if there were no significant color alteration, they would provide a sensation of whiter teeth. In addition, possible side effects caused by whitening dentifrices, such as tooth sensitivity, requires more clinical evidence on the safety and effectiveness of these products.

The hypothesis of our study was accepted, as both whitening dentifrices tested caused clinically perceptible color alteration without causing TS. In this respect, the findings of this clinical trial reveal that even though there was no statistical difference in $\Delta \mathrm{E}$ among the groups, the overall clinical effect showed that all of the dentifrices, including the control one, caused a clinically perceptible color alteration. According to the NBS criteria, the results obtained for $\Delta \mathrm{E}$ after 4 weeks of treatment $\mathrm{G} 1(\Delta \mathrm{E}=5.1)$, $\mathrm{G} 2(\Delta \mathrm{E}=6.8)$ and $\mathrm{G} 3(\Delta \mathrm{E}=4.4)$ are clinically perceptible to the human eye. By evaluating $\Delta \mathrm{E}$, it may be implied that this color alteration is related to an overall reduction in tooth staining.
The main components of a whitening dentifrice include surfactants, polyphosphates, enzymes - some of which contain low peroxide concentrations and abrasives that are probably the primary stain removal ingredient in these dentifrices. ${ }^{6,19} \mathrm{In}$ fact, whitening dentifrices are considered superficial stain agents rather than bleaching agents per $s e,{ }_{1}^{19}$ since abrasive cleaning primarily exerts influence only on extrinsic stains and does not greatly influence any underlying intrinsic discoloration or the natural shade of the tooth. ${ }^{2}$ In our study, the tested dentifrices did not contain hydrogen peroxide in their composition; nevertheless, all of them, including the control one, had abrasive agents. Therefore, it may be suggested that the cause of this color alteration could result from the efficacy of different abrasive agents in the gradual removal of extrinsic stains.

The whitening effect has been associated with the amount of abrasives in dentifrices ${ }^{6}$ and in addition to type, its amount is closely related to dentifrice abrasiveness. ${ }^{4}$ Thus, the color alteration at different 
levels observed in all of the tested dentifrices may be associated with these factors. While dentifrices in G1 and G2 were silica-based, that in G3-control was the only dentifrice that contained calcium carbonate in its composition and was the one with the lowest $\Delta \mathrm{E}$ values. Likewise, improved tooth brushing (frequency and technique) may have influenced the color alteration also observed in the control group (G3) due to the stain removal effect.

As to lightness, $\Delta \mathrm{L}$ showed a significant difference in G1 (Colgate Luminous White) at the second and third assessment points; however, no differences were observed when this group was compared with the other groups. Alterations in lightness have been attributed to the presence of high cleaning silica and of polyphosphates ${ }^{14}$ with removal of extrinsic stains and better results than conventional dentifrices. ${ }^{25}$ Previous clinical studys ${ }^{14}$ has shown the lightening action of Colgate Luminous White, but no noticeably visible change after 15 days of use. In our study, although color alteration after 4 weeks was clinically perceptible, the difference in luminosity was only observed in the time factor.

With respect to the $b^{*}$ parameter, only G2 (Close Up White Now) showed significantly different $\Delta \mathrm{b}$ results (baseline-4weeks) when compared to G1 and control. Over time, $\Delta \mathrm{b}$ was significantly higher after 4 weeks than in the first 3 weeks. In this regard, Close Up White Now contains blue covarine as active agent, which alters the optical properties of the teeth by changing the $b^{*}$ parameter from yellow to blue, with an overall improvement in perceivable tooth whitening. ${ }^{26}$ According to Joiner, ${ }^{2}$ silica-based dentifrices containing blue covarine are not only effective in removing extrinsic stains, but also in significantly whitening the intrinsic color of teeth. Probably, the results obtained in $\Delta \mathrm{b}$ may have been influenced by color alteration results, which had the highest values in $\mathrm{G} 2$ since $\Delta \mathrm{E}$ corresponds to luminosity (a* and $\mathrm{b}^{*}$ parameters). ${ }^{16}$

Even though abrasive agents have an important role in cleaning teeth, ${ }^{21}$ in high amounts, they may lead to increased wear and roughness of enamel and dentin, especially when combined with at-home or in-office bleaching treatments, ${ }^{20,21}$ altering restorations by accelerating their degradation and causing biofilm retention. ${ }^{22,27}$ In addition, previous clinical studies have reported tooth sensitivity and oral irritation as side effects in response to peroxide-containing whitening dentifrices, ${ }^{23}$ usually when associated with erosive forces. ${ }^{28}$

In the present study, TS was also evaluated and, in spite of the abrasive effect, there was no significant $(p>0.05)$ increase in TS considering the baseline values for G1, G2 or G3. Probably, the results found in our study may be associated with peroxide-free whitening dentifrices. Moreover, the source of fluoride $(1,100-1,450 \mathrm{ppm})$ contained in all tested dentifrices has been demonstrated to be effective in reducing enamel solubility and in promoting its remineralization ${ }^{29}$ and, consequently, avoiding TS.

On the other hand, it is important to mention some limitations of this study. The age of the participants (younger subjects) who are more interested in having whitened teeth, the renewed interest in tooth brushing, and the improved behavior of participants who are aware of being observed ('Hawthorn effect') ${ }^{30}$ may have influenced the results. Noteworthy, the participants included in this study had good oral hygiene and no conditions that could cause TS. Considering that tooth brushing is a daily procedure, further studies will be needed to corroborate these findings, especially when associated with other procedures, such as bleaching. Furthermore, it would be important to design long-term clinical trials with these types of products to observe for how long they can be safe and effective. Taking into account the limitations of this study, our findings show that the tested dentifrices were capable of altering tooth color after 4 weeks of use without causing TS.

\section{Conclusion}

The tested whitening and control dentifrices showed a similar and clinically perceptible performance in tooth color change $(\Delta \mathrm{E})$ without causing significant $\mathrm{TS}$.

\section{Acknowledgments}

The authors are grateful to $\mathrm{CNPq}$ (National Council of Technological and Scientific Development) for the financial support. The authors declare no potential conflicts of interest with respect to the publication of this article. 


\section{References}

1. Alkhatib MN, Holt R, Bedi R. Age and perception of dental appearance and tooth colour. Gerodontology. 2005;22(1):326. doi:10.1111/j.1741-2358.2004.00045.x

2. Joiner A. Whitening toothpastes: a review of the literature. J Dent. 2010;38 Suppl 2:e17-24. doi:10.1016/j.jdent.2010.05.017

3. Meireles SS, Goettems ML, Dantas RV, Bona AD, Santos IS, Demarco FF. Changes in oral health related quality of life after dental bleaching in a double-blind randomized clinical trial. J Dent. 2014;42(2):114-21. doi:10.1016/j.jdent.2013.11.022

4. Lima DA, Silva AL, Aguiar FH, Liporoni PC, Munin E, Ambrosano GM et al. In vitro assessment of the effectiveness of whitening dentifrices for the removal of extrinsic tooth stains. Braz Oral Res. 2008;22(2):106-11. doi:10.1590/S1806-83242008000200003

5. Ghassemi A, Hooper W, Vorwerk L, Domke T, DeSciscio $\mathrm{P}$, Nathoo S. Effectiveness of a new dentifrice with baking soda and peroxide in removing extrinsic stain and whitening teeth. J Clin Dent. 2012;23(3):86-91.

6. Alshara S, Lippert F, Eckert GJ, Hara AT. Effectiveness and mode of action of whitening dentifrices on enamel extrinsic stains. Clin Oral Investig. 2014;18(2):563-9. doi:10.1007/s00784-013-0981-8

7. Ayad F, Demarchi B, Khalaf A, Petrone ME, Chaknis P, DeVizio $W$ et al. A six-week clinical efficacy study of a new dentifrice for the removal of extrinsic tooth stain. J Clin Dent. 1999;10(3 Spec No):103-6.

8. Yankell SL, Emling RC, Petrone ME, Rustogi K, Volpe AR, DeVizio W et al. A six-week clinical efficacy study of four commercially available dentifrices for the removal of extrinsic tooth stain. J Clin Dent. 1999;10(3 Spec No):115-8.

9. Nathoo S, Petrone ME, DeVizio W, Chaknis P, Volpe AR. A six-week clinical study to compare the stain removal efficacy of three dentifrices. J Clin Dent. 2002;13(2):91-4.

10. Singh S, Mankodi S, Chaknis P, Petrone ME, DeVizio $\mathrm{W}$, Volpe AR et al. The clinical efficacy of a new tooth whitening dentifrice formulation: a six-month study in adults. J Clin Dent. 2002;13(2):86-90.

11. Walsh TF, Rawlinson A, Wildgoose D, Marlow I, Haywood J, Ward JM. Clinical evaluation of the stain removing ability of a whitening dentifrice and stain controlling system. J Dent. 2005 May;33(5):413-8. doi:10.1016/j.jdent.2004.10.021

12. Hotwan, K, Thosar N, Baliga S. Comparative in vitro assessment of color stability of hybrid esthetic restorative materials against various children's beverages. J Conserv Dent. 2014;17(1):70-4. doi:10.4103/0972-0707.124154

13. Torres CR, Perote LC, Gutierrez NC, Pucci CR, Borges AB. Efficacy of mouth rinses and toothpaste on tooth whitening. Oper Dent. 2013;38(1):57-62. doi:10.2341/11-360-L.

14. Horn BA, Bittencourt BF, Gomes OM, Farhat PA. Clinical evaluation of the whitening effect of over-the-counter dentifrices on vital teeth. Braz Dent J. 2014;25(3):203-6. doi:10.1590/0103-6440201300053
15. Revised American Dental Association specification no. 12 for denture base polymers. J Am Dent Assoc. 1975 Feb;90(2):451-8. doi:10.14219/jada.archive.1975.0069

16. Pirolo R, Mondelli RF, Correr GM, Gonzaga CC, Furuse AY. Effect of coffee and a cola-based soft drink on the color stability of bleached bovine incisors considering the time elapsed after bleaching. J Appl Oral Sci. 2014;22(6):534-40. doi:10.1590/1678-775720130578

17. Carey CM. Tooth whitening: what we now know. J Evid Based Dent Pract. 2014 Jun;14 Suppl:70-6. doi:10.1016/j.jebdp.2014.02.006

18. Menezes M, Turssi CP, Hara AT, Messias DC, Serra MC. Abrasion of eroded root dentine brushed with different toothpastes. Clin Oral Investig. 2004 Sep;8(3):151-5. doi:10.1007/s00784-004-0262-7

19. Demarco FF, Meireles SS, Masotti AS. Over-the-counter whitening agents: a concise review. Braz Oral Res. 2009;23 Suppl 1:64-70. doi:10.1590/S1806-83242009000500010

20. Özkan P, Kansu G, Özak ST, Kurtulmuş-Yilmaz S, Kansu P. Effect of bleaching agents and whitening dentifrices on the surface roughness of human teeth enamel. Acta Odontol Scand. 2013;71(3-4):488-97. doi:10.3109/00016357.2012.696691

21. Hilgenberg SP, Pinto SC, Farago PV, Santos FA, Wambier DS. Physical-chemical characteristics of whitening toothpaste and evaluation of its effects on enamel roughness. Braz Oral Res. 2011;25(4):288-94. doi:10.1590/S1806-83242011005000012

22. Barbieri GM, Mota EG, Rodrigues-Junior SA, Burnett LH. Effect of whitening dentifrices on the surface roughness of commercial composites. J Esthet Restor Dent. 2011;23(5):338-45. doi:10.1111/j.1708-8240.2011.00426.x

23. Gerlach RW, Barker ML, Tucker HL. Clinical response of three whitening products having different peroxide delivery: comparison of tray, paint-on gel, and dentifrice. J Clin Dent. 2004;15(4):112-7.

24. Faul F, Erdfelder E, Lang AG, Buchner A. G*Power 3: a flexible statistical power analysis program for the social, behavioral, and biomedical sciences. Behav Res Methods. 2007;39(2):175-91. doi:10.3758/BF03193146

25. Sharma N, Galustians HJ, Qaqish J, Rustogi K, Zhang YP, Petrone ME et al. Comparative tooth whitening and extrinsic tooth stain prevention efficacy of a new dentifrice and a commercially available tooth whitening dentifrice: six-week clinical trial. J Clin Dent. 2004;15(2):52-7.

26. Joiner A, Philpotts CJ, Alonso C, Ashcroft AT, Sygrove NJ. A novel optical approach to achieving tooth whitening. J Dent. 2008;36 Suppl 1:S8-14. doi:10.1016/j.jdent.2008.02.005

27. Pinto SC, Hilgenberg SP, Wambier DS, Farago PV, Bandéca MC, Santos FA. Characterization of dentifrices containing desensitizing agents, triclosan or whitening agents: EDX and SEM analysis. Braz Dent J. 2014;25(2):153-9. doi:10.1590/0103-6440201302209 
28. West N, Newcombe RG, Hughes N, Mason S, Maggio B, Sufi $\mathrm{F}$, et al. A 3-day randomised clinical study investigating the efficacy of two toothpastes, designed to occlude dentine tubules, for the treatment of dentine hypersensitivity. J Dent. 2013;41(2):187-94. doi:10.1016/j.jdent.2012.11.007

29. Lynch RJ, ten Cate JM. The anti-caries efficacy of calcium carbonate-based fluoride toothpastes. Int Dent J. 2005;55(3 Suppl 1):175-8. doi:10.1111/j.1875-595X.2005.tb00055.x

30. McCambridge J, Witton J, Elbourne DR. Systematic review of the Hawthorne effect: new concepts are needed to study research participation effects. J Clin Epidemiol. 2014 Mar;67(3):267-77. doi:10.1016/j.jclinepi.2013.08.015 